\title{
GMR
}

\section{Paracrine effect of bone marrow mesenchymal stem cells on proliferation, apoptosis, and alpha- actin-2 expression in hepatic stellate cells}

\author{
H.-J. Cao' ${ }^{1}$, M.-D. Wang ${ }^{2}$, S.-G. Li ${ }^{1}$, L. Zhu ${ }^{1}$ and J.-H. Zheng ${ }^{1}$ \\ ${ }^{1}$ Department of Gastrointestinal Medicine, \\ The First Affiliated Hospital of Zhejiang Chinese Medical University, \\ Hangzhou, China \\ ${ }^{2}$ Department of Emergency and Trauma Center, \\ The First Affiliated Hospital of Zhejiang Chinese Medical University, \\ Hangzhou, China \\ Corresponding author: M.-D. Wang \\ E-mail: wangmudan_d@163.com \\ Genet. Mol. Res. 16 (1): gmr16019201 \\ Received September 6, 2016 \\ Accepted December 9, 2016 \\ Published March 16, 2017 \\ DOI http://dx.doi.org/10.4238/gmr16019201 \\ Copyright $(2017$ The Authors. This is an open-access article distributed under the terms of \\ the Creative Commons Attribution ShareAlike (CC BY-SA) 4.0 License.
}

\begin{abstract}
We investigated the paracrine effects of bone marrow mesenchymal stem cells (BMSCs) on the proliferation, apoptosis, and alpha-actin-2 (ACTA2) expression of hepatic stellate cells (HSCs), and explored the possible mechanisms of hepatocyte growth factor (HGF). We established a co-culture system by culturing BMSCs on the upper layer and HSCs on the lower layer of a 6-well Transwell plate. Normal HSCs were cultured alone as a control. Cell apoptosis was determined by flow cytometry. We detected the expression of ACTA2 mRNA and ACTA2 protein in HSC using quantitative reverse transcription polymerase chain reaction (qRT-PCR) and western blotting, respectively. ACTA2 in HSCs was detected by fluorescent staining, and HGF in the co-culture supernatant was detected by enzyme-linked immunosorbent assay (ELISA). The apoptotic rate of HSCs in the experiment group was 2.6 times that in the control group
\end{abstract}

Genetics and Molecular Research 16 (1): gmr16019201 
$(\mathrm{P}<0.05)$. The expression levels of ACTA2 mRNA and ACTA2 protein were significantly inhibited in HSCs compared with the control group $(\mathrm{P}<0.05)$. HGF concentration in the co-culture supernatant was 0.43 $\pm 0.47 \mathrm{mM}$ in the experimental group, which was significantly higher than in the control group $(0.16 \pm 0.43 \mathrm{mM})(\mathrm{P}<0.05)$. The paracrine effect of BMSCs, which was caused by the suppression of ACTA2 and HGF expression, induced HSC apoptosis.

Key words: Bone marrow mesenchymal stem cell; Hepatic stellate cell; Paracrine; Proliferation; Apoptosis; ACTA2

\section{INTRODUCTION}

Liver cirrhosis is one of the most common diseases that threaten human health, and there is no special treatment for the clinical management of end-stage liver disease (Liou, 2014). Mesenchymal stem cell (MSC) injection treatment is a new therapy that is used to treat liver cirrhosis in MSC transplantation, and the therapeutic effects of the phase I-II clinical trials are inspirational (Kharaziha et al., 2009; El-Ansary et al., 2012).

However, the mechanism underlying MSC treatment is still unclear. Some scientists think that the rate of MSC colonization in the liver is low, with very few MSCs transforming into functional cells (Anjos-Afonso et al., 2004; Kuroda et al., 2011). The therapeutic benefits of the treatment have been attributed to the paracrine effects of MSCs (van Poll et al., 2008; Nagaishi et al., 2014). Liver fibrosis is the first stage of liver cirrhosis, and the activation of hepatic stellate cells (HSCs) is the main cause of liver fibrosis. The excessive secretion of collagen by activated HSCs induces hyperplasia and deposition of extracellular matrix (ECM), which ultimately leads to liver fibrosis and cirrhosis. Therefore, inducing and promoting the apoptosis of HSCs is an efficient liver fibrosis therapy.

We investigated the paracrine effects of MSCs on liver fibrosis using activated HSCs. We also observed the effects of bone marrow mesenchymal stem cells (BMSCs) on the proliferation, apoptosis, and ACTA2 expression of HSCs by co-culture of BMSCs and HSCs on a Transwell plate, and investigated the possible effects of hepatocyte growth factor (HGF).

\section{MATERIAL AND METHODS}

\section{Materials}

The bone marrow was obtained from healthy volunteers at the First Affiliated Hospital, Zhejiang University. This study was approved by the Ethics Committee of the First Affiliated Hospital of Nan Jing Medical University. Informed consent was obtained from all the volunteers. HSC-T6 and fibroblast cell lines were obtained from the Sun Yat-Sen University Cancer Center. An Annexin V-fluorescein isothiocyanate (FITC)/propidium iodide (PI) kit and a protein extraction kit were obtained from Keygen Biotech Co., Ltd. (Nanjing, China). SYBR Green fluorescent dyes were obtained from Takara Biotechnology Co., Ltd. (Dalian, China). An MDA detecting kit was obtained from Nanjing Jiancheng Bioengineering Institute (China). HyClone cell culture medium [comprising Dulbecco's modified Eagle's medium (DMEM), fetal bovine serum (FBS), and trypsin] was obtained from Thermo Fisher Scientific

Genetics and Molecular Research 16 (1): gmr16019201 
Inc in USA. (Thermo Fisher Scientific, Shanghai, China). Mouse anti-human ACTA2 and mouse anti-human $\beta$-actin antibodies were obtained from Santa Cruz Biotechnology, Inc. (Shanghai, China). TRIzol was obtained from Invitrogen (Thermo Fisher Scientific Inc.). The reverse transcription polymerase chain reaction (RT-PCR) kit was from MBI Fermentas (Beijing, China); the electrochemiluminescence (ECL) kit was from Thermo Fisher Scientific Inc.; the polyvinylidene diffuoride (PVDF) membrane was from Merck Millipore Corporation (Shanghai, China); and the Transwell insert semipermeable membrane was from Corning Costar (Shanghai, China).

\section{Methods}

\section{Separation and culture of BMSCs}

Human bone marrow $(5 \mathrm{~mL})$ was centrifuged to separate the mononuclear cells, and 1 x $10^{6}$ cells were seeded onto a $10-\mathrm{cm}$ culture dish. After $24 \mathrm{~h}$, the culture medium was replaced with DMEM containing $10 \%$ FBS and culture was resumed. When confluence reached $80 \%$, the BMSCs were digested with trypsin containing $0.25 \%$ ethylenediaminetetraacetic acid (EDTA) to produce a subculture with a density of $1 \times 10^{5} / \mathrm{mL}$. The third passage was used for the experiment.

\section{HSC culture}

The HSCs were cultured in DMEM containing 10\% FBS. When confluence reached $80 \%$, the HSCs were digested with trypsin containing $0.05 \%$ EDTA to produce a subculture.

\section{Co-culture of BMSCs with HSCs}

The Transwell plate comprised two layers with a $0.4-\mu \mathrm{m}$ semipermeable membrane between them. The cells in the two layers were not permitted to come into contact with each other. The BMSCs were cultured in the insert chamber of the Transwell plate to a density of 1-2 x $10^{5}$ per well. The same density of HSCs was cultured in the lower layer. DMEM $(1.5 \mathrm{~mL})$ containing $10 \%$ FBS was added to each well. When adherence was complete, the culture medium was replaced with fresh medium to construct the Transwell co-culture system. The BMSCs were co-cultured with HSCs in the experimental group, and the control group comprised HSCs on their own.

\section{Apoptosis detection}

An Annexin V-FITC/PI kit was used to detect the apoptosis of HSCs. The cells were fixed, incubated, and stained in the dark, and apoptosis was detected by flow cytometry. The early apoptotic cells were labeled Annexin $\mathrm{V}^{+} / \mathrm{PI}^{-}$, whereas the advanced apoptotic cells were labeled Annexin $\mathrm{V}^{+} / \mathrm{PI}^{+}$. The apoptosis rate was the sum of the two rates mentioned above. There were three multiple wells in each group. The experiment was repeated three times.

\section{Determination of HGF concentration by ELISA}

Apart from the blank control, the supernatant of the BMSCs and the different 
concentrations of standard sample $(100 \mu \mathrm{L} /$ well $)$ were combined and incubated at $37^{\circ} \mathrm{C}$ for 90 $\min$. The cells were then washed five times and the working solution of biotinylated antibody $(100 \mu \mathrm{L} /$ well $)$ was added. The cells were then incubated at $37^{\circ} \mathrm{C}$ for $60 \mathrm{~min}$. The cells were washed four times and the enzyme conjugate working solution $(100 \mu \mathrm{L} /$ well $)$ was added. The cells were then incubated at $37^{\circ} \mathrm{C}$ for $30 \mathrm{~min}$. The cells were washed five times and the chromogenic reagent $(100 \mu \mathrm{L} /$ well $)$ was added. The cells were incubated at $37^{\circ} \mathrm{C}$ for 15 $20 \mathrm{~min}$ in the dark. The final solution $(100 \mu \mathrm{L} /$ well $)$ was mixed and HGF was immediately investigated using the enzyme mark instrument at $\mathrm{A}_{450}$.

\section{Detection of ACTA2 mRNA by quantitative reverse transcription polymerase chain reaction (qRT-PCR)}

According to the specification, RNA was extracted and reverse-transcribed into complementary DNA (cDNA). The cDNA $(2 \mu \mathrm{L})$ was used as a template for amplifying ACTA2. The total volume of the PCR system was $20 \mu \mathrm{L}$. A $440 \mathrm{bp}$ amplified fragment of ACTA2 was obtained using the forward primer $5^{\prime}$-TTTUTCUTCUUTTAUCUT-3' and the reverse primer 5'-TTTUTCUTCUUTTAUCUT-3'. A 191 bp amplified fragment of $\beta$-actin was used as an internal control using the forward primer 5'-AACUACCCCTTCATTUAC-3' and the reverse primer 5'-TCCACUACATACTCAUCAC-3'. The PCR conditions were as follows: $95^{\circ} \mathrm{C}$ for $30 \mathrm{~s}, 95^{\circ} \mathrm{C}$ for $5 \mathrm{~s}, 60^{\circ} \mathrm{C}$ for $30 \mathrm{~s}$, for a total of 40 cycles. The $2^{-\Delta \Delta \mathrm{Ct}}$ method was used for analysis of $A C T A 2$ expression. Three multiple wells per primer were used and the average result was calculated. Three multiple pores were present in each group. The experiment was repeated three times.

\section{Detection of the ACTA2 protein by western blotting}

Protein samples were collected from the experimental and control groups, and their concentrations were determined using the BCA method. The protein samples were separated by sodium dodecyl sulfate polyacrylamide gel electrophoresis with a $5 \%$ stacking gel and a 10\% separating gel. The proteins were then transferred to PVDF membranes, which were blocked with $5 \%$ non-fat milk at $25^{\circ} \mathrm{C}$ for $2 \mathrm{~h}$. Primary antibodies (ACTA2 1:200, $\beta$-actin 1:500) were added and incubated at $4^{\circ} \mathrm{C}$ overnight. Secondary antibodies $(1: 10,000)$ were added and incubated at $25^{\circ} \mathrm{C}$ for $1 \mathrm{~h}$. ECL was used to visualize the samples.

\section{Cellular immunofluorescence (IF)}

The ACTA2 in BMSCs was detected using a CM-H2DCFDA probe. According to the specification, the working solution of CM-H2DCFDA had a final concentration of $5 \mu \mathrm{M}$. The working solution $(1 \mathrm{~mL})$ was added to each well followed by incubation at $37^{\circ} \mathrm{C}$ for $30 \mathrm{~min}$. The staining results were detected at $488 \mathrm{~nm}$ by fluorescence microscopy.

\section{Statistical analysis}

All the measured data are reported as means \pm standard deviation. The $t$-test was used to compare groups. A P value $<0.05$ was considered a significant difference.

Genetics and Molecular Research 16 (1): gmr16019201 


\section{RESULTS}

\section{BMSC separation, culture, and identification}

Because of the differences in adherence between BMSCs and other kinds of cells, the volume and digesting time were controlled as follows. Trypsin $(2 \mathrm{~mL})$ was added to the 10-cm plate, which was incubated at $37^{\circ} \mathrm{C}$ for $3 \mathrm{~min}$, with the total digesting time less than $5 \mathrm{~min}$. After 2 days, the strip cells grew and were distributed sparsely. After the 7th day, MSC clusters appeared, proliferated, and extended. The BMSCs were spindle-shaped or fusiform, and grew with vorticity. The BMSCs were arranged closely and had homogeneous morphology (Figure 1).
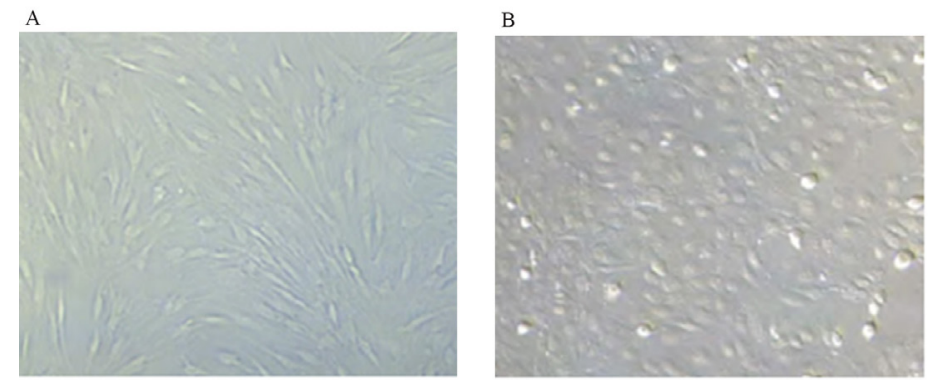

Figure 1. A. Third passage of bone marrow mesenchymal stem cells (BMSCs) (100X). B. Third passage of hepatic stellate cells (HSCs) (100X).

\section{Apoptosis}

The apoptotic rate of HSCs was $(6.95 \pm 0.61) \%$ in the experimental group, which was 2.6 times that in the control group $[(2.64 \pm 0.66) \% ; \mathrm{P}<0.05)]$ (Figure 2$)$.
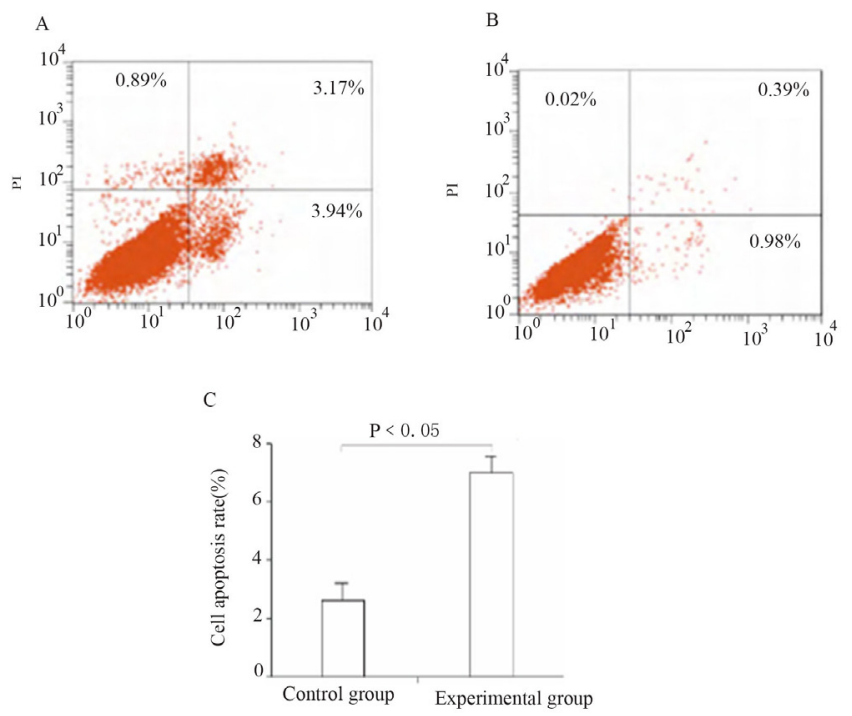

Figure 2. A. Experimental group. B. Control group. C. Apoptotic rate (\%).

Genetics and Molecular Research 16 (1): gmr16019201 


\section{ACTA2 expression}

ACTA2 mRNA expression was $0.75 \pm 0.15$ in the experimental group, which was less than $1.62 \pm 0.10$ that in the control group $(\mathrm{P}<0.05)$ (Figure $3 \mathrm{~A})$. This result indicates that the secretion in the supernatant of BMSCs could inhibit ACTA2 mRNA expression in HSCs. The results of western blotting showed that ACTA2 protein expression was significantly lower in the experimental group than in the control group (Figure 3B).

A

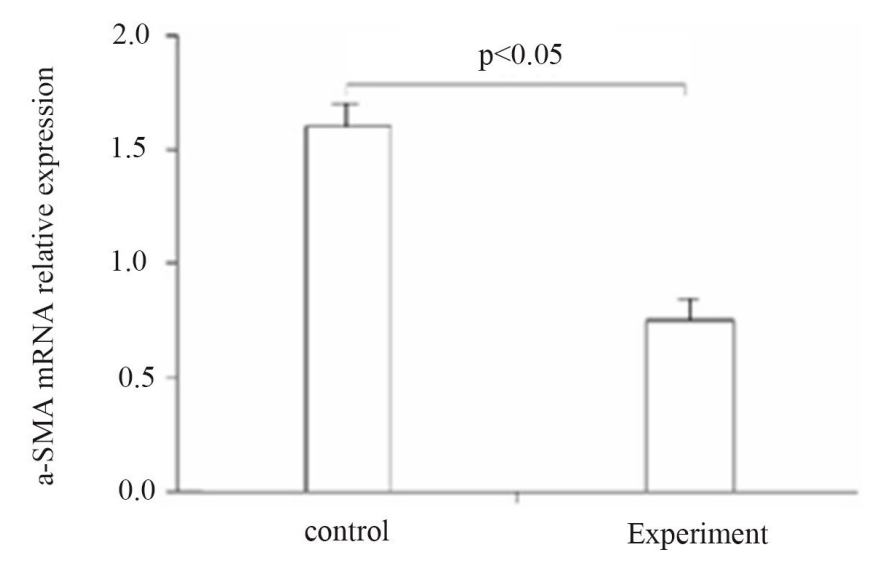

B

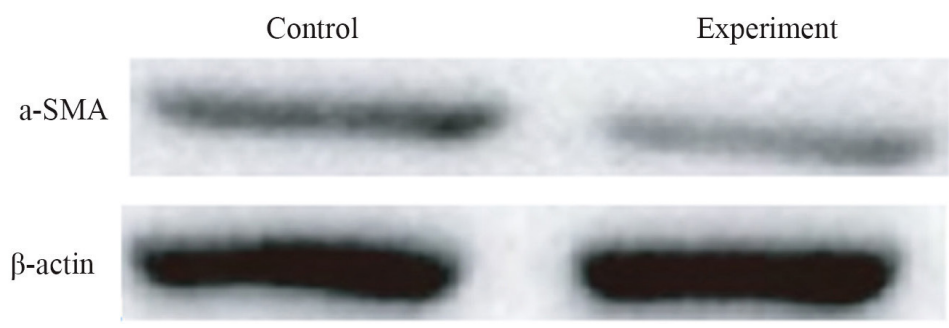

Figure 3. A. ACTA2 mRNA expression in hepatic stellate cells (HSCs). B. ACTA2 protein expression in hepatic stellate cells (HSCs).

\section{ACTA2 expression in HSCs by IF}

ACTA2 expression was higher in the experimental group, as shown by the IF signal (arrow) of ACTA2 at the junctions of the cell (Figure 4A). ACTA2 expression was lower in the control group, with the IF signal of ACTA2 at the edge and junctions of the cell (Figure 4B).

\section{HGF concentration}

After the co-culture of BMSCs with HSCs, the HGF concentration in the experimental group was 2.5 times that in the control group. There was a significant difference between the two groups $(\mathrm{P}<0.05)$ (Figure 5).

Genetics and Molecular Research 16 (1): gmr16019201 

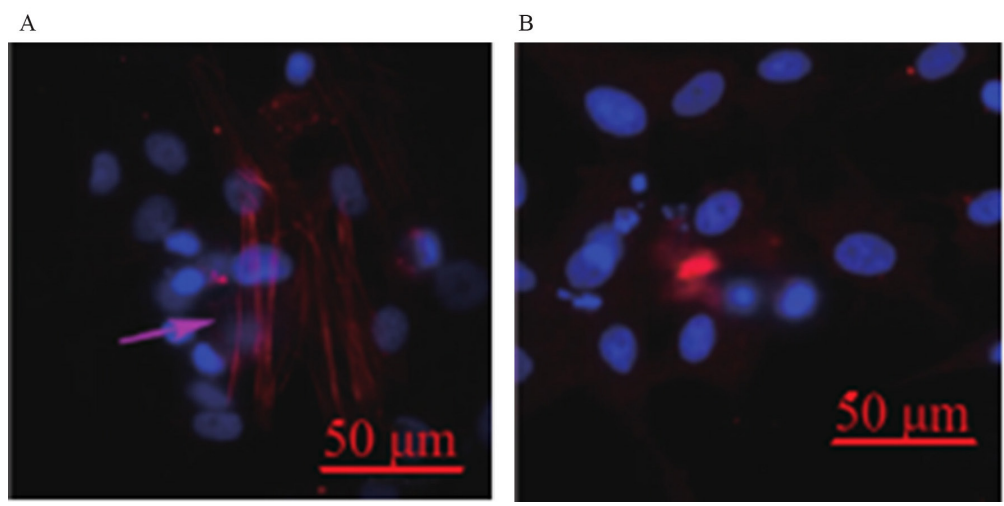

Figure 4. A. Immunofluorescence of ACTA2 in the experimental group. B. Immunofluorescence of ACTA2 in the control group.

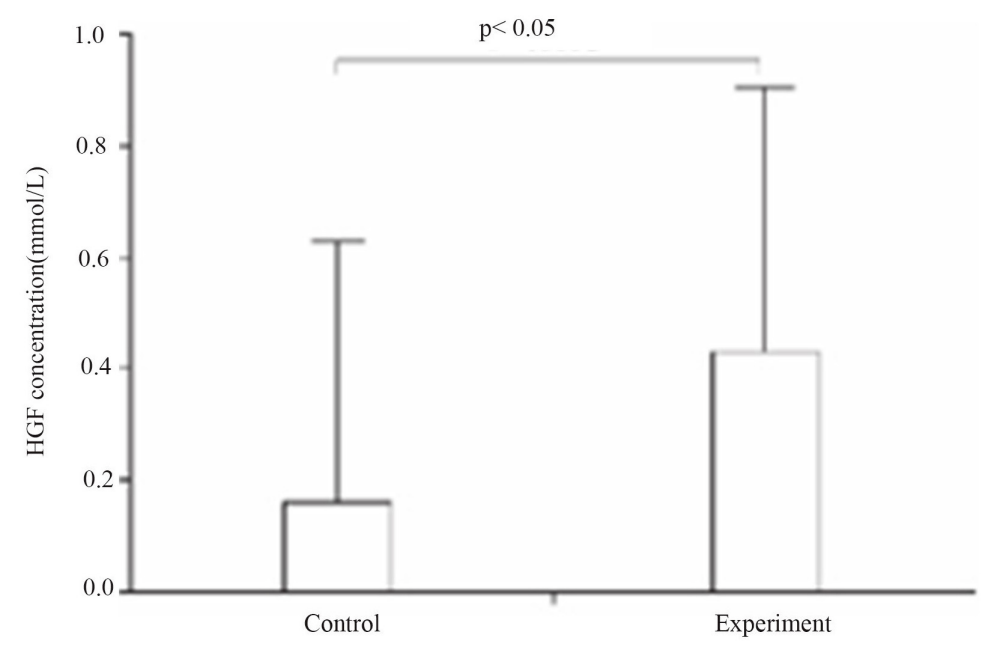

Figure 5. Hepatocyte growth factor (HGF) concentration in the supernatant (mM).

\section{DISCUSSION}

Liver fibrosis has a very complicated mechanism and the critical fibrotic process involves the activation of HSCs. The activated HSCs secrete excessive amounts of ACTA2 and synthesize extracellular matrix, which leads to the over-accumulation of extracellular matrix. Therefore, the activation and proliferation of HSCs is important in the pathogenesis of liver fibrosis. HSCs are the principle target cells for therapeutic purposes (Li et al., 2007), and ACTA2, which is the special marker of activated HSCs, is widely used in studies on liver fibrosis because the expression level of ACTA2 is closely related to the level of fibrosis ( $\mathrm{Li}$ et al., 2009).

Recently, the therapeutic benefits of MSC paracrine effects have attracted increasing attention. In vitro, the MSC paracrine effect induces many cytokines, growth factors, and 
inflammatory mediators, which can regulate apoptosis, proliferation, and inflammatory reactions, etc. (Khubutiya et al., 2014). Fouraschen et al. (2012) injected the supernatant of cultured MSCs into an animal model and resected most of the liver; they found that the liver regenerated and liver function was recovered, which indicates that the MSC paracrine material contained something with a therapeutic effect. A previous study by Chen et al. (2010) showed that HGF from MSC paracrine material can inhibit HSC proliferation and induce HSC apoptosis by the HGF/c-met signal, RohA, etc.

To determine whether BMSCs can regulate HSC proliferation, apoptosis, and ACTA2 expression, we used activated HSCs to simulate liver fibrosis. We co-cultured BMSCs with HSCs in a ratio of 1:1 to construct a Transwell system. The results from the co-culturing system showed that BMSCs could inhibit HSC proliferation, promote HSC apoptosis, and indirectly suppress ACTA2 expression. We hypothesized that something in the co-culturing system was inhibiting HSC proliferation, promoting HSC apoptosis, and suppressing ACTA2 expression. We then determined the HGF concentration in the supernatant after co-culturing for $48 \mathrm{~h}$; the results showed that the HGF concentration was significantly higher in the experimental group than in the control group. These results were in accord with the theory that HGF is the main factor for promoting HSC apoptosis (Parekkadan et al., 2007). However, another study (Kendall et al., 2009) has shown that nerve growth factor is the important factor in the BMSC paracrine effect. Therefore, we think that HGF plays an important role in inhibiting HSC proliferation in the BMSC paracrine effect, and promotes HSC apoptosis and suppresses ACTA2 expression.

In conclusion, co-culture of BMSCs with HSCs in the Transwell system inhibited the proliferation of HSCs and promoted the apoptosis of HSCs. ACTA2 expression was suppressed and HGF played an important role in the mechanism. These results might indicate the mechanism underlying the therapeutic effects of BMSC transplantation in the treatment of acute and chronic liver injury, as well as the molecular mechanism of the anti-fibrosis effect shown by BMSCs. However, there were several limitations to this study and we will further confirm the results in vivo.

\section{Conflicts of interest}

The authors declare no conflict of interest.

\section{ACKNOWLEDGMENTS}

Research supported by the Zhejiang Provincial Natural Science Foundation of China with Grant \#Y2100379.

\section{REFERENCES}

Anjos-Afonso F, Siapati EK and Bonnet D (2004). In vivo contribution of murine mesenchymal stem cells into multiple cell-types under minimal damage conditions. J. Cell Sci. 117: 5655-5664. http://dx.doi.org/10.1242/jcs.01488

Chen GZ, Jiang HX, Lu ZF and Xiao J (2010). Mesenchymal stem cells co-cultured hepatic stellate cell proliferation, apoptosis and regulate the expression of RohA. World Chin. J. Digestology 18: 1643-1649.

El-Ansary M, Abdel-Aziz I, Mogawer S, Abdel-Hamid S, et al. (2012). Phase II trial: undifferentiated versus differentiated autologous mesenchymal stem cells transplantation in Egyptian patients with HCV induced liver cirrhosis. Stem Cell Rev. 8: 972-981. http://dx.doi.org/10.1007/s12015-011-9322-y

Genetics and Molecular Research 16 (1): gmr16019201 
Fouraschen SM, Pan Q, de Ruiter PE, Farid WR, et al. (2012). Secreted factors of human liver-derived mesenchymal stem cells promote liver regeneration early after partial hepatectomy. Stem Cells Dev. 21: 2410-2419. http://dx.doi. org $/ 10.1089 / \mathrm{scd} .2011 .0560$

Kendall TJ, Hennedige S, Aucott RL, Hartland SN, et al. (2009). p75 Neurotrophin receptor signaling regulates hepatic myofibroblast proliferation and apoptosis in recovery from rodent liver fibrosis. Hepatology 49: 901-910. http:// dx.doi.org/10.1002/hep.22701

Kharaziha P, Hellström PM, Noorinayer B, Farzaneh F, et al. (2009). Improvement of liver function in liver cirrhosis patients after autologous mesenchymal stem cell injection: a phase I-II clinical trial. Eur. J. Gastroenterol. Hepato1. 21: 1199-1205.

Khubutiya MS, Vagabov AV, Temnov AA and Sklifas AN (2014). Paracrine mechanisms of proliferative, anti-apoptotic and anti-inflammatory effects of mesenchymal stromal cells in models of acute organ injury. Cytotherapy 16: 579585. http://dx.doi.org/10.1016/j.jcyt.2013.07.017

Kuroda Y, Kitada M, Wakao S and Dezawa M (2011). Bone marrow mesenchymal cells: how do they contribute to tissue repair and are they really stem cells? Arch. Immunol. Ther. Exp. (Warsz.) 59: 369-378. http://dx.doi.org/10.1007/ s00005-011-0139-9

Li TT, Wang H, Liao CX and Ping J (2007). Activation of hepatic stellate cells and the molecular mechanism of liver fibrosis treatment. Journal of Wuhan University 28: 256-261.

Li ZG, Yang JX, Zhang W, Wang ZM, et al. (2009). Hepatic Xinning human rat liver fibrosis a-SMA, MMP-13 and TIMP-1 protein expression. Journal of Beijing University of Traditional Chinese Medicine. Clin. Med. (Northfield Ill.) 16: 6-9.

Liou IW (2014). Management of end-stage liver disease. Med. Clin. North Am. 98: 119-152. http://dx.doi.org/10.1016/j. mena.2013.09.006

Nagaishi K, Ataka K, Echizen E, Arimura Y, et al. (2014). Mesenchymal stem cell therapy ameliorates diabetic hepatocyte damage in mice by inhibiting infiltration of bone marrow-derived cells. Hepatology 59: 1816-1829. http://dx.doi. org $/ 10.1002 /$ hep. 26975

Parekkadan B, van Poll D, Megeed Z, Kobayashi N, et al. (2007). Immunomodulation of activated hepatic stellate cells by mesenchymal stem cells. Biochem. Biophys. Res. Commun. 363: 247-252. http://dx.doi.org/10.1016/j. bbrc.2007.05.150

van Poll D, Parekkadan B, Cho CH, Berthiaume F, et al. (2008). Mesenchymal stem cell-derived molecules directly modulate hepatocellular death and regeneration in vitro and in vivo. Hepatology 47: 1634-1643. http://dx.doi. org $/ 10.1002 /$ hep. 22236

Genetics and Molecular Research 16 (1): gmr16019201 